\title{
Differential immune cell infiltrations between healthy periodontal and chronic periodontitis tissues
}

Wei Li ${ }^{1}$, Zheng Zhang ${ }^{2^{*}}$ and Zuo-min Wang ${ }^{1 *}$

\begin{abstract}
Background: Host immunity plays an important role against oral microorganisms in periodontitis.

Methods: This study assessed the infiltrating immune cell subtypes in 133 healthy periodontal and 210 chronic periodontitis tissues from Gene Expression Omnibus (GEO) datasets using the CIBERSORT gene signature files.

Results: Plasma cells, naive B cells and neutrophils were all elevated in periodontitis tissues, when compared to those in healthy controls. In contrast, memory B cells, resting dendritic, mast cells and CD4 memory cells, as well as activated mast cells, M1 and M2 macrophages, and follicular helper T cells, were mainly present in healthy periodontal tissues. Furthermore, these periodontitis tissues generally contained a higher proportion of activated CD4 memory T cells, while the other subtypes of T cells, including resting CD4 memory T cells, CD8 T cells, follicular helper T cells $\left(T_{F H}\right)$ and regulatory $T$ cells (Tregs), were relatively lower in periodontitis tissues, when compared to healthy tissues. The ratio of dendritic and mast cells and macrophages was lower in periodontitis tissues, when compared to healthy tissues. In addition, there was a significant negative association of plasma cells with most of the other immune cells, such as plasma cells vs. memory B cells $(\gamma=-0.84)$, plasma cells vs, resting dendritic cells $(\gamma=-0.64)$, plasma cells vs. resting CD4 memory T cells $(\gamma=0.50)$, plasma cells versus activated dendritic cells $(\gamma=-0.46)$, plasma cells versus $T_{F H}(\gamma=-0.46)$, plasma cells versus macrophage $M 2$ cells $(\gamma=-0.43)$, or plasma cells versus macrophage $M 1$ cells $(\gamma=-0.40)$, between healthy control and periodontitis tissues.
\end{abstract}

Conclusion: Plasma cells, naive B cells and neutrophils were all elevated in periodontitis tissues. The infiltration of different immune cell subtypes in the periodontitis site could lead the host immunity against periodontitis.

Keywords: Periodontitis, Host immunity, Inflammation, Infiltrating immune cells and subtypes

\section{Background}

Periodontitis is a chronic inflammatory disease in the surrounding tooth tissues [1] characterized by periodontal plaque microorganism-induced inflammation and loss of periodontal attachment [2]. This occurs in approximately $10-15 \%$ of the global population as the

\footnotetext{
*Correspondence: zhangzheng@nankai.edu.cn; wzuomin@sina.cn ${ }^{1}$ Department of Stomatology, Beijing Chao-Yang Hospital, Capital Medical University, 8th Gongti South Road, Beijing 100020, China

${ }^{2}$ Department of Periodontology, Tianjin Stomatological Hospital, Tianjin Key Laboratory of Oral Function Reconstruction, Hospital of Stomatology, Nankai University, 75th Dagu North Road, Tianjin 300000, China
}

second main cause of tooth loss among adults [3, 4]. In the early stage of the disease, periodontal supporting tissues present as swollen, red, and/or bleeding (signs of acute inflammation), while in the later stage of the disease, the gums would draw back from the teeth, leading to jaw bone atrophy and tooth loss, and present with acute inflammation symptoms [2-4]. Plaque is the most important initial factor in periodontitis onset. However, plaque alone may not be enough to cause damage to the host periodontal tissue. This is caused by the host's excessive immune response to plaque. Host immunity plays an important role against oral microorganisms,

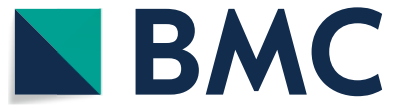

(c) The Author(s) 2020. Open Access This article is licensed under a Creative Commons Attribution 4.0 International License, which permits use, sharing, adaptation, distribution and reproduction in any medium or format, as long as you give appropriate credit to the original author(s) and the source, provide a link to the Creative Commons licence, and indicate if changes were made. The images or other third party material in this article are included in the article's Creative Commons licence, unless indicated otherwise in a credit line to the material. If material is not included in the article's Creative Commons licence and your intended use is not permitted by statutory regulation or exceeds the permitted use, you will need to obtain permission directly from the copyright holder. To view a copy of this licence, visit http://creativecommons.org/licenses/by/4.0/. The Creative Commons Public Domain Dedication waiver (http://creativeco mmons.org/publicdomain/zero/1.0/) applies to the data made available in this article, unless otherwise stated in a credit line to the data. 
which is mainly through neutrophil, macrophage, and $\mathrm{T}$ and B-lymphocytes, in order to produce various cytokines and chemokines, and in turn, maintain the tissue homeostasis in the oral cavity [5-8]. To date, the precise molecular mechanisms of oral microorganisminduced disease or protection remains poorly understood [9]. For example, even single and low-abundance species of microorganisms can alter the host-microbial homeostasis to induce an inflammatory disease [10]. Furthermore, Curtis et al. reported that Porphyromonas gingivalis is a keystone pathogen of periodontitis [11]. The maintenance and restoration of oral tissue homeostasis after exposure to pathogens are essential to conquer oral inflammation, and the former depends on the complex coordination of innate and adaptive immune responses. In this regard, the evaluation and identification of tissue-specific immune cell types can help to illustrate the local immunoreactivity and severity of the inflammation. For example, a previous study investigated the development of chronic gingivitis. It was revealed that there was a decrease in fibroblasts (57-39\%), and an increase in plasma cells $(0.2-10.0 \%)$, while the portion of lymphocytes and macrophages remained stable [12]. To date, immunohistochemistry and flow cytometry are the common methodologies for the subtyping of immune cells in tissues, but these do possess some limitations [13]. Thus, the newly developed CIBERSORT technique would allow for the profiling and subtyping of immune cells in tissue specimens for gene expression profiles [13-15]. CIBERSORT is a method developed by Newman et al. [16] to analyze and characterize cell types in complex tissues using their gene expression profiles. Thus, in the present study, the investigators utilized the publically accessible Gene Expression Omnibus (GEO) web data, and applied this for the original CIBERSORT gene signature file [17-20], which profiled and analyzed the different immune cell subtypes between 133 healthy human periodontal tissues and 210 chronic periodontitis tissues. It is expected that the present study would provide useful information regarding the immune cell subpopulations in periodontitis, which could lead to the future control or prevention of periodontitis.

\section{Methods}

\section{Database and data acquisition}

In the present study, the investigators searched the GEO database (https://www.ncbi.nlm.nih.gov/geo/) using the following keywords: "periodontitis," "patient," "gingival tissues," and "gene expression". A total of four microarray datasets were obtained: GSE10334, GSE16134, GSE23586 and GSE54710 [17-20]. Then, the investigators retrieved the basic information of these datasets, but excluded two datasets (GSE23586 and GSE54710) (The reasons were because GSE54710 was a microRNA microarray dataset, which is not the focus of the study, and GSE23586 only contained a very small sample size $[n=6])$. Thus, in the present study, the investigators included two datasets (GSE10334 and GSE16134) for the subsequent data analyses by downloading both GSE10334 [17] and GSE16134 [18] datasets from https://www.ncbi.nlm.nih.gov/geo/ query/acc.cgi?acc=GSE10334 and https://www.ncbi.nlm. nih.gov/geo/query/acc.cgi?acc=GSE16134, respectively. Next, the investigators manually organized the expression profiles of each sample and the corresponding clinical data to obtain the data on the public available gene expression profiles. Each patient in the database contained 2-3 pieces of inflamed gingival tissues for periodontitis tissues $(n=210)$ and human healthy periodontal tissues $(n=133)$. The investigators took the average value of these multiple pieces of tissues for the data calculation and data analysis in the present study.

\section{Evaluation of infiltrating immune cells in periodontal tissues}

Next, the investigators applied the CIBERSORT gene signature file LM22, which contained 22 subtypes of the infiltrating immune cell gene profile, according to a previous study [16], in order to profile and analyze the immune cell subtypes in 133 healthy human periodontal tissues and 210 chronic periodontitis tissues. Specifically, the differentially expressed gene datasets were analyzed with the standard annotation in the CIBERSORT website (https://cibersort.stanford.edu/) using the algorithm run with the default signature matrix at 1000 permutations. The reached CIBERSORT-derived $P$-value using the Monte Carlo sampling provided a confidence measure for each of the generated results.

\section{Statistical analysis}

A total of 22 immune cell subtypes were identified and evaluated using the CIBERSORT metrics. Then, these were statistically analyzed using the Pearson correlation coefficient test. Each sample was quantified for the CIBERSORT-derived $P$-value and root mean squared error (RMSE). Afterwards, the profile of immune cells and the mean value for each tissue type were calculated for each sample, that is, the total macrophage proportion was divided into M0, M1 and M2, while $\mathrm{T}$ cells were classified into $\mathrm{CD} 8+\mathrm{T}$ cells, $\mathrm{CD} 4+$ naive $\mathrm{T}$ cells, $\mathrm{CD} 4+$ memory resting $\mathrm{T}$ cells, $\mathrm{CD} 4+$ memory activated $\mathrm{T}$ cells, follicular helper $\mathrm{T}$ cells, regulatory $\mathrm{T}$ cells (Tregs), and $\mathrm{T}$ cells gamma delta. Student's $t$-test was performed to analyze the difference between two types of tissue samples, while Pearson's correlation coefficient test was used to analyze the 
other immune cell types. All statistical analyses were conducted using the SPSS 13.0 software (SPSS, Chicago, IL, USA).

\section{Results}

\section{Baseline characteristics of the datasets}

The present study obtained and analyzed the differentially expressed gene profiles between human healthy periodontal tissues $(n=133)$ and periodontitis tissues $(n=210)$, and applied the CIBERSORT gene signature file LM22 [16] to assess the different immune cell proportions in 133 healthy human periodontal tissues and 210 chronic periodontitis tissues. Briefly, these tissue samples were taken from 210 non-smokers with periodontitis, and each contributed to a $\geq 2$ "diseased" interproximal papillae (with bleeding on probing [BoP], probing pocket depth $[\mathrm{PPD}] \geq 4 \mathrm{~mm}$, and clinical attachment loss [CAL] of $\geq 3 \mathrm{~mm}$ ), or "healthy" papilla (no BoP; PPD of $\leq 4 \mathrm{~mm}$ and CAL of $\leq 2 \mathrm{~mm}$ ), when such data was available (Table 1). However, merely the GSE10334 dataset provided the patientspecific clinicopathological parameters for a mean age of 42 years old, and 28 teeth, PD $3.9 \mathrm{~mm}$, AL $4.1 \mathrm{~mm}$ and $71 \%$ BoP. Among these gingival tissues, $67 \%$ had a PD of $\geq 5 \mathrm{~mm}$, while $62 \%$ had an AL of $\geq 5 \mathrm{~mm}$ [17].

\section{Distribution of tissue-infiltrated immune cell subtypes in the two databases}

Next, the investigators initially compared the data between these two datasets, and found that the composition ratio of the immune cell subtypes in the periodontitis or control group was similar (Fig. 1a, b and d). Specifically, the plasma and naive B cells and neutrophils were all elevated in the periodontitis group, when compared to those for healthy controls (Fig. 1), while memory B cells, resting dendritic, mast and CD4 memory cells, as well as activated mast cells, M1 and M2 macrophages, and follicular helper $\mathrm{T}$ cells, were higher in healthy periodontal tissues versus periodontitis tissues (Fig. 1c and e).

\section{The changes in immune cells subtypes in healthy and inflammatory periodontal tissues after merging the two databases}

Adaptive immune cells were initially assessed in chronic periodontitis, and the present data revealed that the plasma cell fraction was higher in periodontitis tissues, when compared to that in healthy periodontal tissues (Fig. 2a), while memory B cells were mainly present in healthy periodontal tissues, but less frequent in periodontitis tissues (Fig. 2d). Furthermore, naive B cells were barely visible in healthy periodontal tissues (Fig. 2c), and most of the $\mathrm{T}$ cells that infiltrated the healthy gums were resting memory $\mathrm{T}$ cells, while periodontitis tissues generally contained a higher proportion

Table 1 Characteristics of the individual GEO database data

\begin{tabular}{|c|c|c|}
\hline GEO gene set ID & GSE10334 & GSE16134 \\
\hline Platform & \multicolumn{2}{|c|}{ GPL570: Affymetrix Human Genome U133 plus 2.0 Array } \\
\hline Number of subjects (control versus periodontitis) & 64 versus 90 & 69 versus 120 \\
\hline \multicolumn{3}{|l|}{ Clinical data } \\
\hline Healthy control & $\mathrm{PD} \leq 4 \mathrm{~mm}, \mathrm{CAL} \leq 2 \mathrm{~mm}, \mathrm{BoP}(-)$ & $\mathrm{PD} \leq 4 \mathrm{~mm}, \mathrm{CAL} \leq 2 \mathrm{~mm}, \mathrm{BoP}(-)$ \\
\hline Chronic periodontitis & $\mathrm{PD}>4 \mathrm{~mm}, \mathrm{CAL} \geq 3 \mathrm{~mm}, \mathrm{BoP}(+)$ & $\mathrm{PD}>4 \mathrm{~mm}, \mathrm{CAL} \geq 3 \mathrm{~mm}, \mathrm{BoP}(+)$ \\
\hline$\%$ of sites with bleeding on probing & $71 \pm 0.2$ & Unknown \\
\hline Pocket depth (PD; mm) & $3.9 \pm 0.7$ & Unknown \\
\hline Clinical attachment level (CAL; mm) & $4.1 \pm 0.9$ & Unknown \\
\hline Tissue samples in specified PD range (mm, \%) & & Unknown \\
\hline $1-2$ & 19 & \\
\hline $3-4$ & 14 & \\
\hline 5 & 31 & \\
\hline$\geq 6$ & 36 & \\
\hline Diabetes & Not & Not \\
\hline \multirow[t]{2}{*}{ Smoking } & Not & Not \\
\hline & $\begin{array}{l}\text { No systemic antibiotics or anti-inflammatory } \\
\text { drugs for } \geq 6 \text { months }\end{array}$ & $\begin{array}{l}\text { No systemic antibiotics or } \\
\text { anti-inflammatory drugs } \\
\text { for } \geq 6 \text { months }\end{array}$ \\
\hline PubMed ID & $18,980,520$ & $19,835,625$ \\
\hline
\end{tabular}




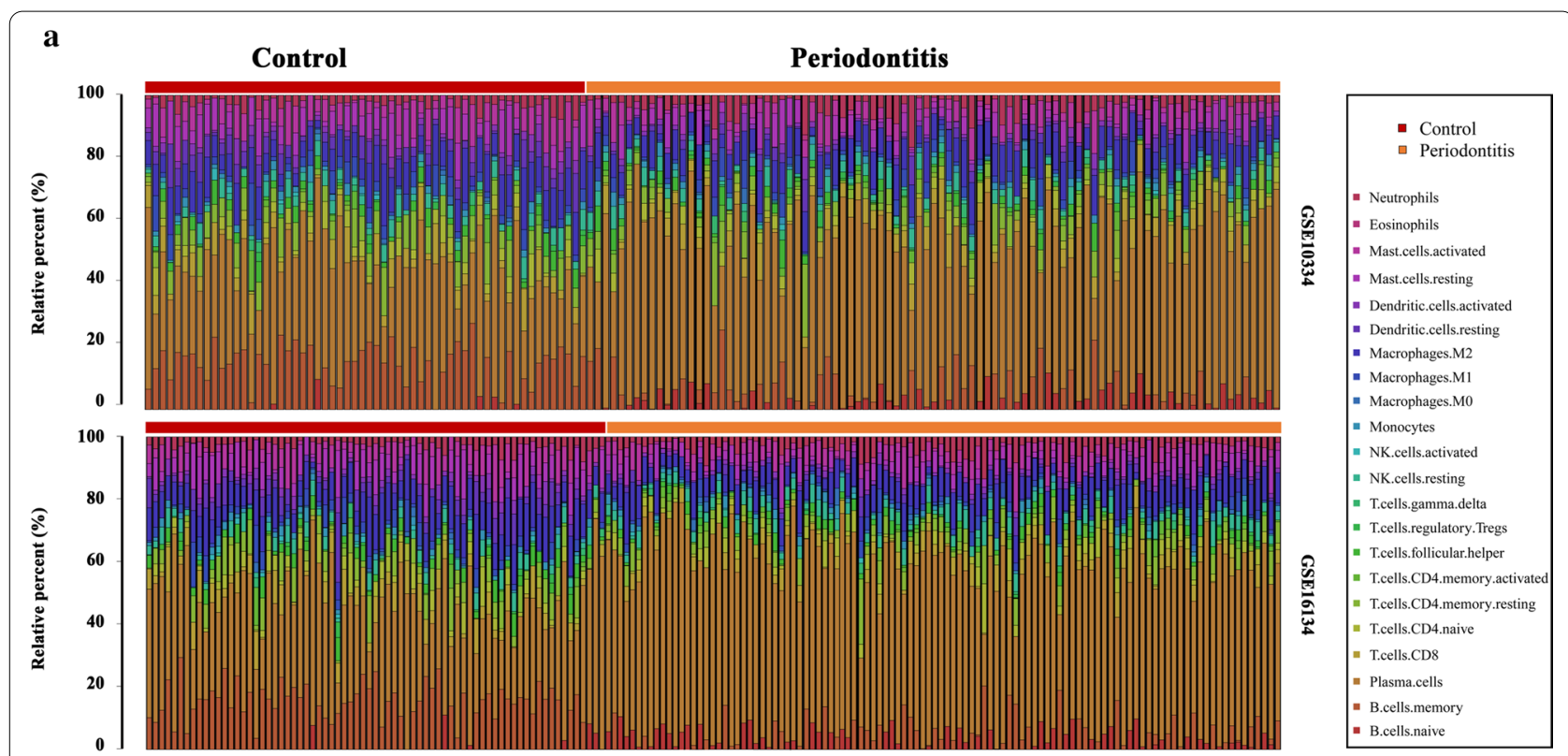

b

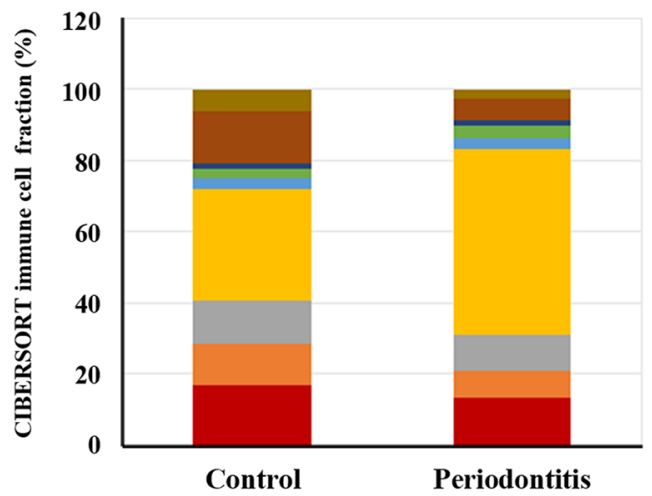

d

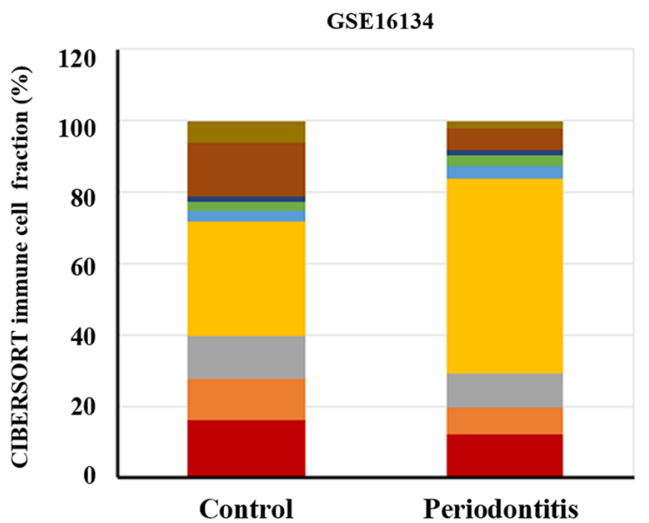

- $\mathrm{T}$ cells

Mast cells

Macrophages

$\square$ Plasma cells

$\square$ NK cells

匹 Neutrophils

- Monocytes

- B cells

- Eosinophils

— Dendritic cells

- $\mathrm{T}$ cells

- Mast cells

- Macrophages

- Plasma cells

- NK cells

- Neutrophils

- Monocytes

- B cells

- Eosinophils

- Dendritic cells c

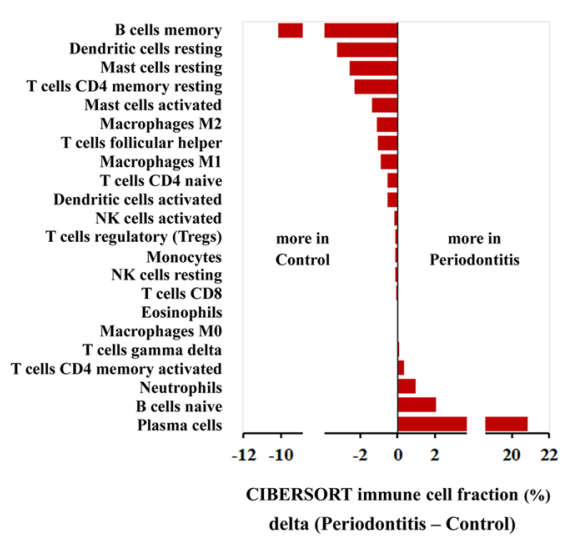

e

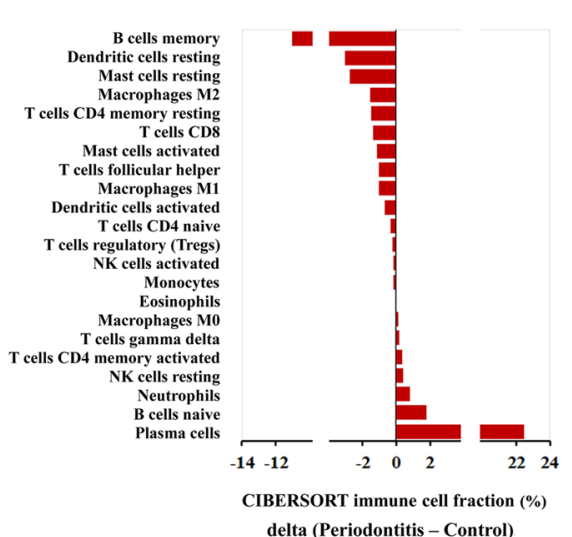

Fig. 1 Differential level of immune cells in each healthy and chronic periodontitis sample. a The different colors and bar lengths indicate the levels of immune cell populations from the two databases. b-e The immune cell composition in the chronic periodontitis and control group in these two databases 


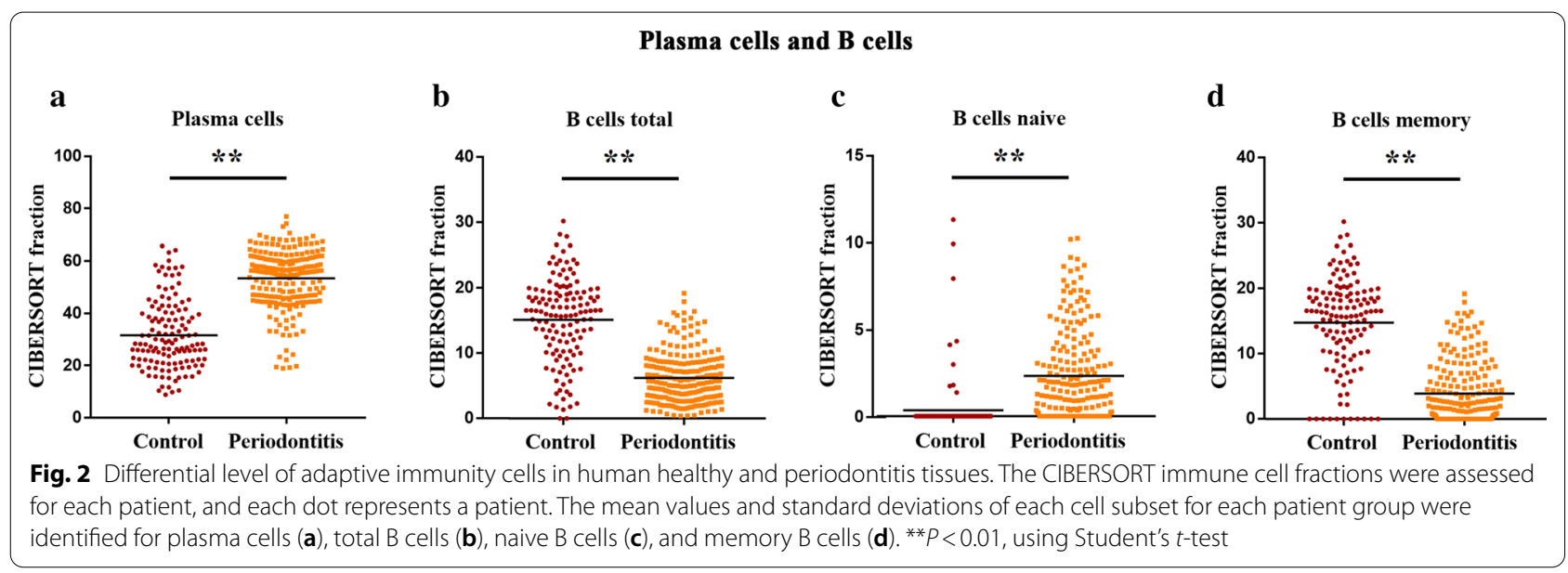

of activated CD4 memory T cells. However, the other subtypes of $\mathrm{T}$ cells, including resting $\mathrm{CD} 4$ memory $\mathrm{T}$ cells, CD8 $\mathrm{T}$ cells, follicular helper $\mathrm{T}$ cells and regulatory $\mathrm{T}$ cells (Tregs), were relatively lower in periodontitis tissues, when compared to those in healthy tissues (Fig. 3). Furthermore, it was found that although the infiltration of these immune cells was higher, when compared to healthy control tissues, the proportion of different cell subtypes varied, and the ratio of dendritic and mast cells, including both the resting and activated ones, and macrophages in periodontitis tissues was lower vs. healthy tissues (Figs. 4 and 5). Neutrophils were the only innate immune cells that increased in the inflamed gingival tissues (Figs. 4 and 5).

\section{T cells}

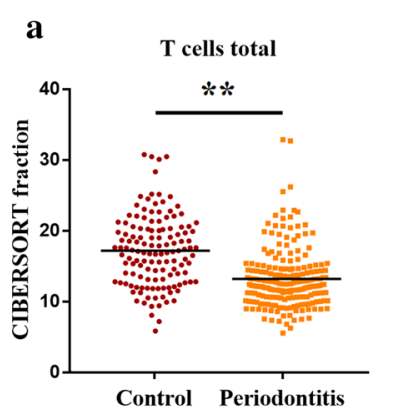

b T cells CD4 memory activated

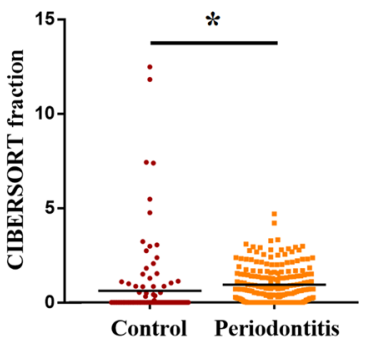

e

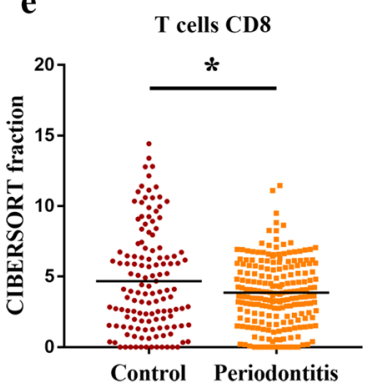

f

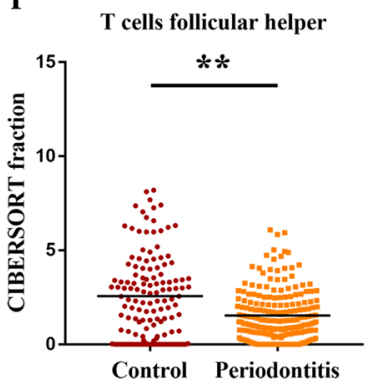

c

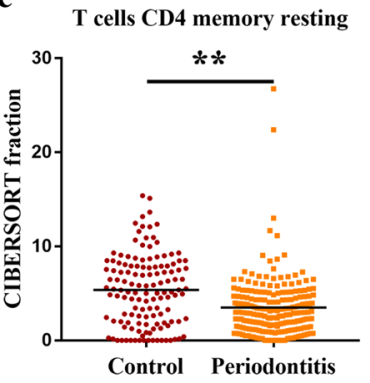

g

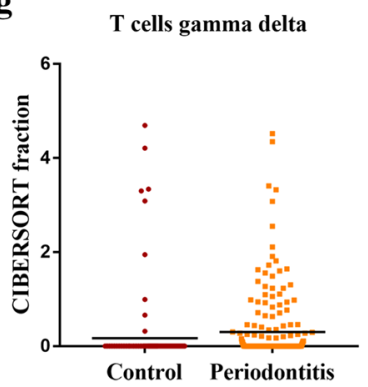

d

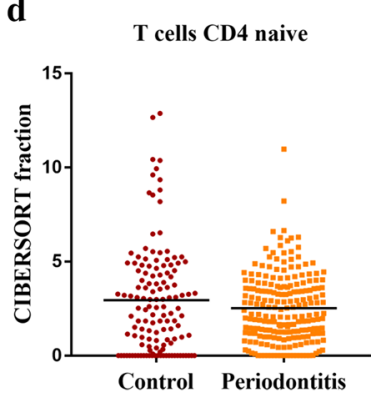

h

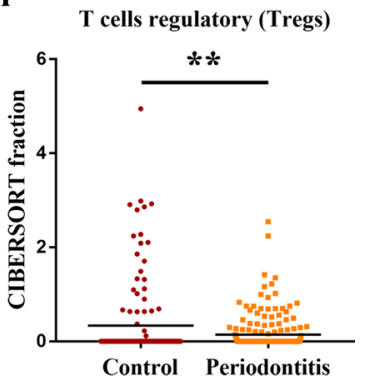

Fig. 3 The differential level of T cell subtypes between human healthy and periodontitis tissues. The CIBERSORT immune cell fractions were determined for each patient, and each dot represents a patient. The mean values and standard deviations for each cell subset for each patient group were identified for the total T cells (a), CD4 memory activated cells (b), CD4 memory resting cells (c), CD4 naive cells (d), CD8 cells (e), follicular helper T cells (TFH) (f), T cells gamma delta ( $\mathbf{g})$ and Tregs $(\mathbf{h}){ }^{*} P<0.05$ and ${ }^{*} P<0.01$, using Student's $t$-test 
Macrophages and NK cells
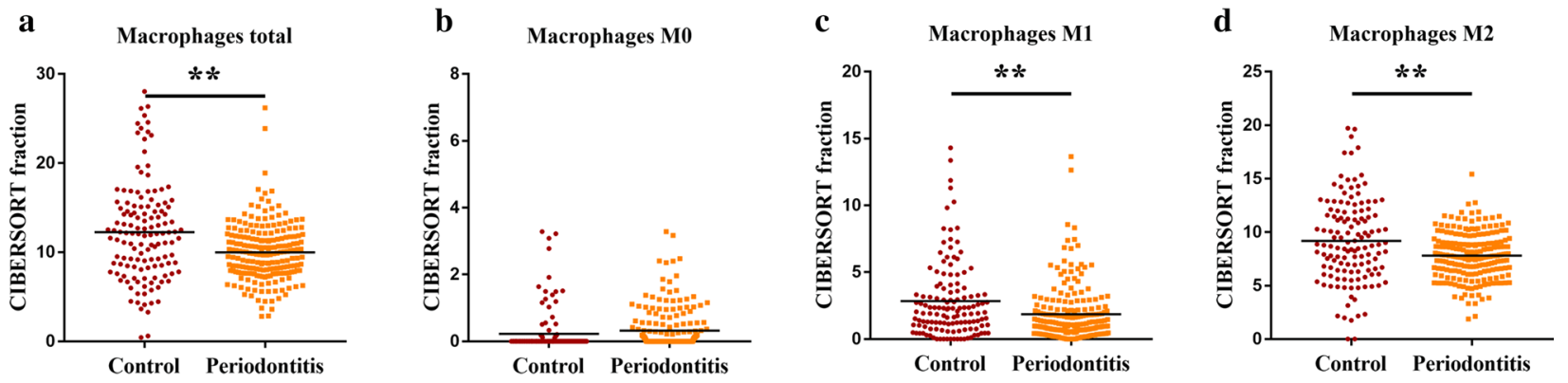

e Macrophages M1/M2 ratio
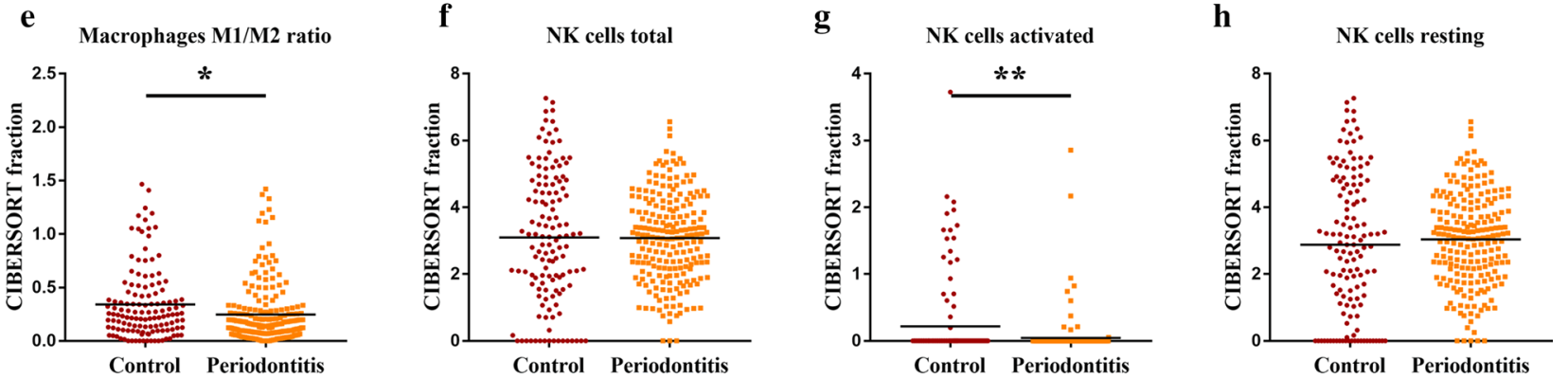

Fig. 4 The characteristics of the differential macrophage and NK cell subpopulations between human healthy and periodontitis tissues. The CIBERSORT immune cell fractions were determined for each patient, and each dot represents a patient. The mean values and standard deviations for each cell subtype of each patient were identified for total macrophages (a), M0 macrophages (b), M1 macrophages (c), M2 macrophages (d), M1/M2 ratio (e), total NK cells (f), resting NK cells $(\mathbf{g})$, and activated NK cells $(\mathbf{h}) .{ }^{*} P<0.05$ and ${ }^{* *} P<0.01$, using Student's $t$-test

\section{Correlation matrix of all 22 immune cell subtypes in periodontitis tissues}

It was found that there was a significant inverse association between plasma cells and most of the other immune cells, that is, plasma cells versus memory B cells $(\gamma=-0.84)$, plasma cells versus resting dendritic cells $(\gamma=-0.64)$, plasma cells versus resting CD4 memory $\mathrm{T}$ cells $(\gamma=0.50)$, plasma cells versus activated dendritic cells $(\gamma=-0.46)$, plasma cells $v s$. $\mathrm{T}_{\mathrm{FH}}(\gamma=-0.46)$, plasma cells versus macrophage M2 cells $(\gamma=-0.43)$, or plasma cells $v s$. macrophage M1 cells $(\gamma=-0.40)$, in periodontitis tissues (Fig. 6). These data indicate that plasma cells might play a central role in the regulation of host immunity against periodontitis. It is possible that plasma cells replace dendritic cells, especially resting dendritic cells, and play a certain role in antigen presentation during periodontitis.

\section{Discussion}

In the present study, the investigators profiled and identified different subtypes of infiltrating immune cells between chronic periodontitis and healthy periodontal tissues. The present data revealed that the plasma and naive B cells and neutrophils were elevated in periodontitis tissues, when compared to those in the healthy control group, while memory B cells, resting dendritic, mast and CD4 memory cells, as well as activated mast cells, M1 and M2 macrophages, and follicular helper $\mathrm{T}$ cells, were mainly present in healthy periodontal tissues. Furthermore, periodontitis tissues contained a higher proportion of activated CD4 memory $\mathrm{T}$ cells, but the other subtypes of $\mathrm{T}$ cells, including resting CD4 memory $\mathrm{T}$ cells, CD8 T cells, follicular helper $\mathrm{T}$ cells, and regulatory $\mathrm{T}$ cells (Tregs), were relatively lower in periodontitis $v s$. healthy tissues. The ratio of dendritic and mast cells and macrophages was lower in periodontitis tissues, when compared to healthy tissues. This led to the significant negative association of plasma cells with memory B cells, resting dendritic cells, resting CD4 memory $\mathrm{T}$ cells, activated dendritic cells, M1 macrophage, M2 macrophage, or $\mathrm{T}_{\mathrm{FH}}$ between healthy controls and periodontitis tissues. In conclusion, the present present data demonstrates that different immune cells play different roles in periodontitis. Specifically, plasma cells might play a central role in the regulation of host immunity against periodontitis, while dendritic cells play a certain role in antigen presentation for the host immunity against periodontitis. 


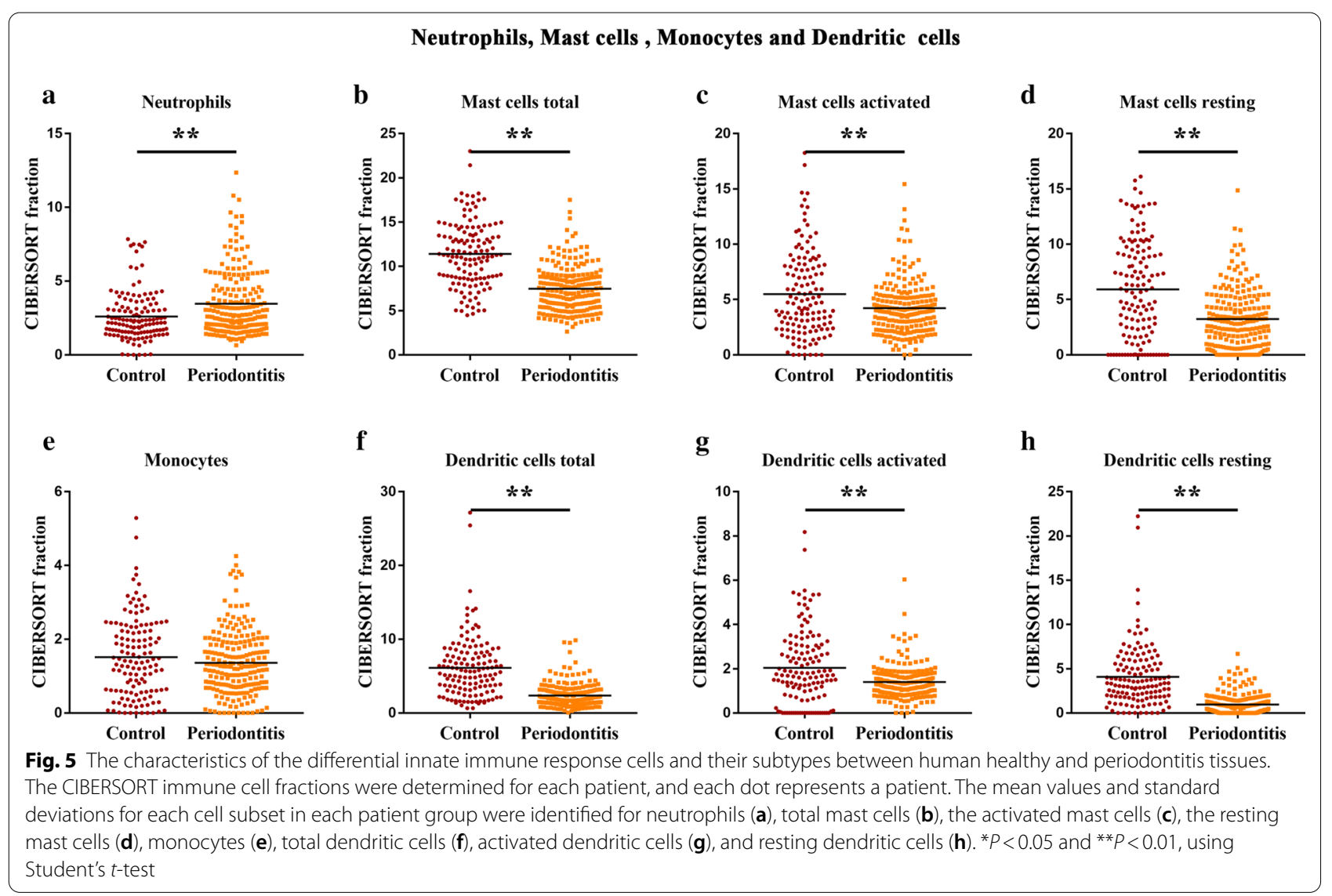

It has been well documented that immune cells possess both inflammation-promoting and -inhibiting roles in tissues. Consistently, it was found that periodontitis tissues have a marked infiltration of different immune cell subtypes, which may be significantly associated with disease development and progression [21, 22]. For example, in parallel to the increase in bacterial exposure and inflammation from healthy to periodontitis, the immune system constantly patrols and provides the surveillance of the gingival environment, and plays a pivotal role in the mediation of local immunity and maintenance of tissue homeostasis [23]. An early previous study assessed the histologic change in the gingiva during six months of abolished oral hygiene to investigate the chronic gingivitis development. The data revealed a decrease in fibroblasts, and an increase in plasma cells, while other cell types, such as lymphocytes and macrophages, remained stable, indicating the host immune responses to gingivitis development [12]. Furthermore, in order to identify these various subtypes of immune cells in tissues, different methodologies, such as immunohistochemistry and flow cytometry, which use specific antibodies, have been used, as reported in a literature [22]. However, these techniques might maximally utilize two antibodies to subtype the immune cells, which is surely not sufficient [13]. Nevertheless, gene profiles and the CIBERSORT gene signature could have more advantages in profiling and identifying the subtypes of immune cells in tissue specimens [13-15]. Thus, the present study profiled immune cell subtypes between healthy controls and periodontitis tissues using a novel CIBERSORT technology, which was previously published in the field of cancer research $[14,24]$. It was observed that plasma, and $B$ and $T$ cells were the main immune cells in healthy periodontal tissues, while plasma cells were the major immune cells in periodontal tissues obtained from periodontitis patients, but the proportion of other types of immune cells was reduced.

A previous study revealed that the B cell lineage was the predominant cell type in periodontitis, while for plasma cells, the effector cells differentiated from B cells, which accounted for approximately $50-60 \%$ of the total infiltrating immune cells in periodontitis tissues [25]. The present data surly further confirms this previous study [25]. Indeed, previous studies have reported the production of a disease-specific antibody in the 1980 s, which were conducted by Czerkinsky's group [26-28] and Holt's group.[29, 30]. Plasma cells have 


\begin{tabular}{|c|c|c|c|c|c|c|c|c|c|c|c|c|c|c|c|c|c|c|c|c|c|c|c|}
\hline & 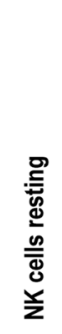 & 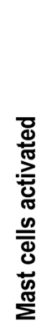 & 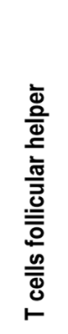 & 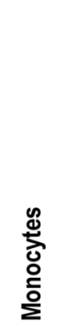 & 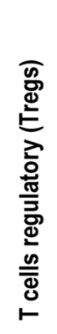 & 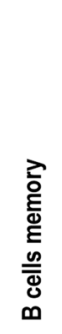 & 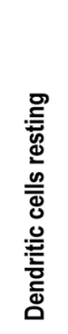 & $\begin{array}{l}\frac{0}{\overline{0}} \\
\frac{0}{0} \\
\frac{0}{2} \\
\frac{0}{2}\end{array}$ & 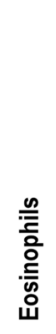 & 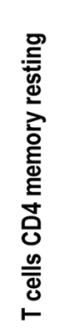 & 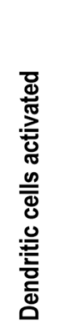 & 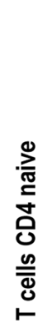 & 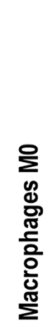 & 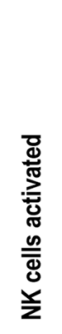 & 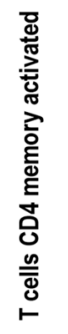 & 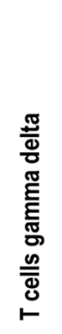 & 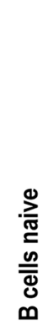 & 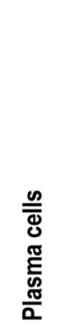 & 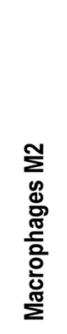 & 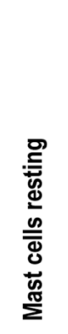 & 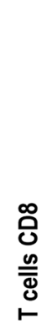 & 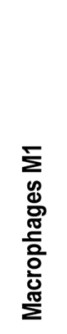 & \\
\hline NK cells resting & 1.00 & 0.33 & -0.06 & 0.26 & 0.05 & $72 \mathrm{e}-0 \mathrm{c}$ & $x-0.13$ & 0.07 & -0.01 & -0.06 & -0.05 & 0.08 & 0.08 & -0.33 & $-0.01-$ & -0.25 & 0.05 & -0.02 & -0.03 & -0.29 & -0.25 & -0.13 & \\
\hline Mast cells activated & 0.33 & 1.00 & 0.20 & 0.16 & -0.01 & 0.36 & 0.17 & 0.16 & 0.02 & 0.15 & 0.36 & 0.05 & -0.21 & -0.19 & $-0.17-$ & -0.09 & -0.19 & -0.33 & -0.02 & -0.46 & -0.13 & -0.19 & \\
\hline$T$ cells follicular helper & -0.06 & 0.20 & 1.00 & 0.28 & 0.25 & 0.45 & 0.37 & -0.11 & 0.02 & 0.07 & 0.33 & -0.20 & -0.11 & 0.26 & $-0.19-$ & -0.01 & -0.11 & -0.46 & 0.01 & 0.13 & -0.02 & 0.05 & 0.8 \\
\hline Monocytes & 0.26 & 0.16 & 0.28 & 1.00 & 0.03 & 0.19 & $.15 \mathrm{e}-0$ & $1-0.29$ & -0.01 & -0.05 & -0.01 & 0.18 & 0.02 & 0.10 & -0.21 & -0.24 & 0.02 & -0.10 & -0.24 & -0.09 & -0.01 & -0.12 & \\
\hline $\mathrm{T}$ cells regulatory (Tregs) & 0.05 & -0.01 & 0.25 & 0.03 & 1.00 & 0.18 & 0.36 & -0.03 & -0.02 & -0.10 & $45 \mathrm{e}-0 \mathrm{c}$ & -0.17 & -0.08 & 0.06 & $-0.14-$ & -0.04 & -0.14 & -0.24 & 0.18 & 0.15 & 0.07 & 0.17 & 0.6 \\
\hline B cells memory & $72 \mathrm{e}-0 \mathrm{c}$ & 0.36 & 0.45 & 0.19 & 0.18 & 1.00 & 0.46 & -0.09 & -0.05 & 0.37 & 0.32 & 0.12 & -0.12 & 0.25 & -0.15 & -0.01 & -0.58 & -0.84 & 0.15 & 0.28 & 0.04 & 0.19 & \\
\hline Dendritic cells resting & -0.13 & 0.17 & 0.37 & $.15 \mathrm{e}-0$ & 10.36 & 0.46 & 1.00 & $40 \mathrm{e}-01$ & 10.06 & 0.26 & 0.32 & -0.12 & -0.18 & 0.22 & $-0.16-$ & -0.04 & -0.36 & -0.64 & 0.23 & 0.33 & 0.14 & 0.24 & \\
\hline Neutrophils & 0.07 & 0.16 & -0.11 & -0.29 & -0.03 & -0.09 & $.40 \mathrm{e}-0$ & 1.00 & 0.11 & 0.19 & 0.20 & -0.16 & -0.13 & -0.05 & -0.03 & 0.09 & -0.05 & -0.04 & 0.10 & -0.14 & -0.14 & -0.18 & 0.4 \\
\hline Eosinophils & -0.01 & 0.02 & 0.02 & -0.01 & -0.02 & -0.05 & 0.06 & 0.11 & 1.00 & 0.14 & 0.12 & -0.03 & -0.02 & -0.01 & -0.03 .0 & $.04 e-0$ & 1-0.02 & -0.01 & -0.01 & -0.06 & -0.02 & -0.04 & \\
\hline T cells CD4 memory resting & -0.06 & 0.15 & 0.07 & -0.05 & -0.10 & 0.37 & 0.26 & 0.19 & 0.14 & 1.00 & 0.31 & -0.05 & -0.04 & 0.19 & 0.04 & 0.14 & -0.24 & -0.50 & 0.13 & 0.13 & -0.31 & 0.11 & 0.2 \\
\hline Dendritic cells activated & -0.05 & 0.36 & 0.33 & -0.014 & $45 \mathrm{e}-0 \mathrm{c}$ & $(0.32$ & 0.32 & 0.20 & 0.12 & 0.31 & 1.00 & 0.02 & -0.11 & 0.01 & 0.07 & 0.15 & -0.18 & -0.46 & 0.15 & -0.07 & 0.03 & -0.16 & \\
\hline T cells CD4 naive & 0.08 & 0.05 & -0.20 & 0.18 & -0.17 & 0.12 & -0.12 & -0.16 & -0.03 & -0.05 & 0.02 & 1.00 & 0.04 & -0.09 & -0.01 & 0.05 & -0.13 & -0.09 & 0.03 & -0.06 & -0.21 & $18 \mathrm{e}-0 \mathrm{c}$ & \\
\hline Macrophages MO & 0.08 & -0.21 & -0.11 & 0.02 & -0.08 & -0.12 & -0.18 & -0.13 & -0.02 & -0.04 & -0.11 & 0.04 & 1.00 & -0.02 & 0.31 & 0.09 & 0.03 & 0.04 & -0.02 & 0.03 & 0.09 & 0.21 & \\
\hline NK cells activated & -0.33 & -0.19 & 0.26 & 0.10 & 0.06 & 0.25 & 0.22 & -0.05 & -0.01 & 0.19 & 0.01 & -0.09 & -0.02 & 1.00 & -0.11 & 0.06 & -0.13 & -0.22 & -0.06 & 0.28 & 0.09 & 0.04 & \\
\hline $\mathrm{T}$ cells $\mathrm{CD} 4$ memory activated & -0.01 & -0.17 & -0.19 & -0.21 & -0.14 & -0.15 & -0.16 & -0.03 & -0.03 & 0.04 & 0.07 & -0.01 & 0.31 & -0.11 & $1.00 \mathrm{c}-\mathrm{c} \cdot \mathrm{c}-\mathrm{c}$ & 0.42 & 0.05 & -0.02 & 0.04 & -0.04 & 0.13 & 0.27 & -0.2 \\
\hline T cells gamma delta & -0.25 & -0.09 & -0.01 & -0.24 & -0.04 & -0.01 & -0.04 & 0.09 & $.04 \mathrm{e}-0$ & 0.14 & 0.15 & 0.05 & 0.09 & 0.06 & 0.42 & 1.00 & -0.03 & -0.09 & 0.03 & -0.02 & -0.09 & 0.23 & \\
\hline B cells naive & 0.05 & -0.19 & -0.11 & 0.02 & -0.14 & -0.58 & -0.36 & -0.05 & -0.02 & -0.24 & -0.18 & -0.13 & 0.03 & -0.13 & 0.05 & -0.03 & 1.00 & 0.51 & -0.26 & -0.29 & -0.14 & -0.24 & -0.4 \\
\hline Plasma cells & -0.02 & -0.33 & -0.46 & -0.10 & -0.24 & -0.84 & -0.64 & -0.04 & -0.01 & -0.50 & -0.46 & -0.09 & 0.04 & -0.22 & $-0.02-$ & -0.09 & 0.51 & 1.00 & -0.43 & -0.39 & -0.16 & -0.40 & \\
\hline Macrophages M2 & -0.03 & -0.02 & 0.01 & -0.24 & 0.18 & 0.15 & 0.23 & 0.10 & -0.01 & 0.13 & 0.15 & 0.03 & -0.02 & -0.06 & 0.04 & 0.03 & -0.26 & -0.43 & 1.00 & 0.27 & 0.13 & 0.22 & \\
\hline Mast cells resting & -0.29 & -0.46 & 0.13 & -0.09 & 0.15 & 0.28 & 0.33 & -0.14 & -0.06 & 0.13 & -0.07 & -0.06 & 0.03 & 0.28 & -0.04 & -0.02 & -0.29 & -0.39 & 0.27 & 1.00 & 0.13 & 0.29 & 0.6 \\
\hline T cells CD8 & -0.25 & -0.13 & -0.02 & -0.01 & 0.07 & 0.04 & 0.14 & -0.14 & -0.02 & -0.31 & 0.03 & -0.21 & 0.09 & 0.09 & 0.13 & -0.09 & -0.14 & -0.16 & 0.13 & 0.13 & 1.00 & 0.34 & \\
\hline Macrophages M1 & -0.13 & -0.19 & 0.05 & -0.12 & 0.17 & 0.19 & 0.24 & -0.18 & -0.04 & 0.11 & -0.161 & $18 \mathrm{e}-0 \mathrm{c}$ & $(0.21$ & 0.04 & 0.27 & 0.23 & -0.24 & -0.40 & 0.22 & 0.29 & 0.34 & 1.00 & -0.8 \\
\hline
\end{tabular}

the ability and capacity to produce and release antibodies to conquer the microorganisms in tissues [31], that is, when bacteria invade into the connective tissues of the gum, B cells are activated and become plasma cells for antibody production, and the latter functions as the humoral immunity against periodontal bacteria [32]. Nevertheless, a recent study also demonstrated that plasma cells might also possess the ability to present antigens to $B$ cells, which regulate the acquired humoral immunity through the negative feedback of $\mathrm{T}_{\mathrm{FH}}$ [33]. The present data also confirms this negative feedback, and shows the inverse association of plasma cell level with a variety of immune cells, suggesting that these may have specific immunomodulatory effects in periodontitis development and progression. Furthermore, plasma cells play a key role in immune regulation for the secretion of various cytokines, such as interleukin-10 (IL-10), IL-35, IL-37, granulocytemacrophage colony-stimulating factor (GM-CSF) and inducible nitric oxide synthases (iNOS), in various autoimmune and/or infectious diseases [34, 35]. Certain B cells can suppress antimicrobial immunity by producing IL-35 [34], and compared to healthy tissues, the levels of IL-35 and IL-37 were significantly elevated 
in gingival tissues of chronic periodontitis, indicating that infiltrating plasma cells potentially participated in and regulated the bone loss through IL-35 and IL-37 in periodontitis [36]. Another previous study revealed that plasma cell-produced IL-10 and IL-35 were able to suppress the immunity by acting on myeloid cells and $T$ lymphocytes [37]. In the present study, it was found that the percentage of naive $B$ cells increased, while memory $B$ cells decreased in periodontitis. The functions of memory B cells that reside in human nonlymphoid tissues remain to be defined, and a previous study was the first to report the presence of memory $\mathrm{B}$ cells in healthy gingival tissues [38]. The present study also revealed the highest proportion of memory $\mathrm{B}$ cells in healthy control tissues, when compared to other types of immune cells. Furthermore, in periodontitis, the percentage of memory $B$ cells decreased, and the secreting B cells were higher, when compared to the other subgroups, suggesting their participation in host humoral immunity. Although a previous study revealed the limited involvement of naive B cells in periodontal immune regulation [39], related literatures are relatively rare. The present data revealed that the proportion of naive $B$ cells in periodontitis tissues is elevated, and the role and functions of naive B cells in periodontitis remains unclear. Thus, further studies are needed to understand the involvement in periodontal immune regulation in periodontitis.

Furthermore, the ratio of $\mathrm{T}$ and $\mathrm{B}$ cells also differ among healthy, chronic gingivitis, and adult periodontitis [25]. In innate immunity, neutrophils and various antigen presenting cells would coordinate for the preparation and action of local immunity, and during the course of the disease, the number of infiltrating immune cells would increase [21], but the proportion may differ. For example, neutrophils, as terminally differentiated leukocytes, and the key immune cells for microbial monitoring and innate responses, could accumulate in the acute phase of the disease, playing an important role in the resolution of the inflammation through the release of anti-inflammatory molecules and the organization of phagocytes [23]. In the present study, it was found that neutrophils were elevated, while macrophages and dendritic and mast cells decreased in periodontitis tissues, when compared to healthy control tissues. Furthermore, it was found that there was a high level of M2 macrophages in healthy control tissues. Functionally, macrophages can be divided into M1 and M2 macrophages [40]. M1 macrophages are able to promote the expression of inflammatory factors and bone resorption, while M2 macrophages induce tissue regeneration and repair, such as bone formation, except for the inhibition of inflammation, through the production of anti-inflammatory cytokines [41]. The present data further supports this notion.

However, the present analysis utilized the online database data, and the database lacked some particular clinical information, making it impossible for the investigators to perform the corresponding analysis, such as the analysis of each site severity. In addition, the present study was retrospective study. In order to confirm the present data, future studies with a prospective design are needed.

\section{Conclusion}

The present study profiled and analyzed the subtypes of immune cells between healthy periodontal and chronic periodontitis tissues using online-datasets, which is just a proof-of-principle to identify the high levels of plasma and dendritic cells in periodontitis tissues. This further confirmed that these subtypes of cells could play a central role in the regulation of host immunity against periodontitis. However, further investigations are needed to understand the subtypes of immune cells in the regulation of periodontitis development and progression.

\section{Abbreviations}

BoP: Bleeding on probing; CAL: Clinical attachment loss; GEO: Gene Expression Omnibus; PPD: Probing pocket depth; RMSE: Root mean squared error; $T_{F H}$ : Follicular B helper T cells; Tregs: Regulatory T cells.

\section{Acknowledgements}

The authors would like to thank Prof. Xianliang Li of the Hepatobiliary Surgery Department, Beijing Chao-Yang Hospital, The Capital Medical University

(Beijing, China) for the constructive advice and suggestions.

\section{Authors' contributions}

WL conceived and designed the study. ZZ downloaded the GEO datasets and statistically analyzed and summarized the data, and WL helped in the data analysis and prepared the manuscript. ZW developed the search strategy and reviewed drafts of the paper. All authors read and approved the final version of the manuscript.

\section{Funding}

This work was supported in part by grants from the National Natural Science Foundation of China (\#81870763) and the Natural Science Foundation of Beijing, China (\#7192073).

\section{Availability of data and materials}

The detailed data supporting the present study can be obtained upon reasonable request.

\section{Ethics approval and consent to participation \\ Not applicable.}

\section{Consent for publication}

Not applicable.

\section{Competing interests}

The authors declared that there is no conflict of interest in this work. 
Received: 14 April 2020 Accepted: 18 October 2020

Published online: 27 October 2020

\section{References}

1. Savage A, Eaton KA, Moles DR, Needleman I. A systematic review of definitions of periodontitis and methods that have been used to identify this disease. J Clin Periodontol. 2009;36(6):458-67.

2. Page RC, Offenbacher S, Schroeder HE, Seymour GJ, Kornman KS. Advances in the pathogenesis of periodontitis: summary of developments, clinical implications and future directions. Periodontology. 2000;1997(14):216-48.

3. Albandar JM. Epidemiology and risk factors of periodontal diseases. Dent Clin N Am 2005;49(3):517-32, v-vi.

4. Gross AJ, Paskett KT, Cheever VJ, Lipsky MS. Periodontitis: a global disease and the primary care provider's role. Postgrad Med J. 2017;93(1103):560-5.

5. Ling MR, Chapple IL, Matthews JB. Peripheral blood neutrophil cytokine hyper-reactivity in chronic periodontitis. Innate Immun. 2015;21(7):714-25

6. Abe T, AlSarhan M, Benakanakere MR, Maekawa T, Kinane DF, Cancro MP, et al. The B cell-stimulatory cytokines BLyS and APRIL are elevated in human periodontitis and are required for $B$ cell-dependent bone loss in experimental murine periodontitis. J Immunol. 2015;195(4):1427-35.

7. Zhu LF, Li L, Wang XQ, Pan L, Mei YM, Fu YW, et al. M1 macrophages regulate TLR4/AP1 via paracrine to promote alveolar bone destruction in periodontitis. Oral Dis. 2019;25(8):1972-82.

8. Dutzan N, Kajikawa T, Abusleme L, Greenwell-Wild T, Zuazo CE, Ikeuchi T, et al. A dysbiotic microbiome triggers TH17 cells to mediate oral mucosal immunopathology in mice and humans. Sci Transl Med. 2018;10(463):eaat0797.

9. Hajishengallis G, Darveau RP, Curtis MA. The keystone-pathogen hypothesis. Nat Rev Microbiol. 2012;10(10):717-25.

10. Hajishengallis G, Liang S, Payne MA, Hashim A, Jotwani R, Eskan MA, et al. Low-abundance biofilm species orchestrates inflammatory periodontal disease through the commensal microbiota and complement. Cell Host Microbe. 2011;10(5):497-506.

11. Darveau RP, Hajishengallis G, Curtis MA. Porphyromonas gingivalis as a potential community activist for disease. J Dent Res. 2012;91(9):816-20.

12. Brecx MC, Fröhlicher I, Gehr P, Lang NP. Stereological observations on longterm experimental gingivitis in man. J Clin Periodontol. 1988;15(10):621-7.

13. Chen B, Khodadoust MS, Liu CL, Newman AM, Alizadeh AA. Profiling tumor infiltrating immune cells with CIBERSORT. Methods Mol Biol. 2018;1711:243-59.

14. Rohr-Udilova N, Klinglmuller F, Schulte-Hermann R, Stift J, Herac M, Salzmann $\mathrm{M}$, et al. Deviations of the immune cell landscape between healthy liver and hepatocellular carcinoma. Sci Rep. 2018;8(1):6220.

15. Nirmal AJ, Regan T, Shih BB, Hume DA, Sims AH, Freeman TC. Immune cell gene signatures for profiling the microenvironment of solid tumors. Cancer Immunol Res. 2018;6(11):1388-400.

16. Newman AM, Liu CL, Green MR, Gentles AJ, Feng W, Xu Y, et al. Robust enumeration of cell subsets from tissue expression profiles. Nat Methods. 2015;12(5):453-7.

17. Demmer RT, Behle JH, Wolf DL, Handfield M, Kebschull M, Celenti R, et al. Transcriptomes in healthy and diseased gingival tissues. J Periodontol. 2008;79(11):2112-24.

18. Papapanou PN, Behle JH, Kebschull M, Celenti R, Wolf DL, Handfield M, et al. Subgingival bacterial colonization profiles correlate with gingival tissue gene expression. BMC Microbiol. 2009;9:221.

19. Abe D, Kubota T, Morozumi T, Shimizu T, Nakasone N, Itagaki M, et al. Altered gene expression in leukocyte transendothelial migration and cell communication pathways in periodontitis-affected gingival tissues. J Periodontal Res. 2011:46(3):345-53.

20. Stoecklin-Wasmer C, Guarnieri P, Celenti R, Demmer RT, et al. MicroRNAs and their target genes in gingival tissues. J Dent Res. 2012;91(10):934-40.

21. Zhang Z, Yuan W, Deng J, Wang D, Zhang T, Peng L, et al. Granulocyte colony stimulating factor (G-CSF) regulates neutrophils infiltration and periodontal tissue destruction in an experimental periodontitis. Mol Immunol. 2020;117:110-21.

22. Mahanonda R, Champaiboon C, Subbalekha K, Sa-Ard-lam N, Yongyuth A, Isaraphithakkul B, et al. Memory T cell subsets in healthy gingiva and periodontitis tissues. J Periodontol. 2018;89(9):1121-30.

23. Dutzan N, Konkel JE, Greenwell-Wild T, Moutsopoulos NM. Characterization of the human immune cell network at the gingival barrier. Mucosal Immunol. 2016;9(5):1163-72.
24. Xiong $Y$, Wang $K$, Zhou $H$, Peng L, You W, Fu Z. Profiles of immune infiltration in colorectal cancer and their clinical significant: a gene expression-based study. Cancer Med. 2018;7(9):4496-508.

25. Thorbert-Mros S, Larsson L, Berglundh T. Cellular composition of long-standing gingivitis and periodontitis lesions. J Periodontal Res. 2015;50(4):535-43.

26. Czerkinsky C, Rees AS, Bergmeier LA, Challacombe SJ. The detection and specificity of class specific antibodies to whole bacterial cells using a solid phase radioimmunoassay. Clin Exp Immunol. 1983;53(1):192-200.

27. Czerkinsky C, Prince SJ, Michalek SM, Jackson S, Russell MW, Moldoveanu Z, McGhee JR, Mestecky J. IgA antibody-producing cells in peripheral blood after antigen ingestion: evidence for a common mucosal immune system in humans. Proc Natl Acad Sci U S A. 1987:84(8):2449-53.

28. Czerkinsky C, Nilsson LA, Ouchterlony O, Tarkowski A, Gretzer C. Detection of single antibody-secreting cells generated after in vitro antigen-induced stimulation of human peripheral blood lymphocytes. Scand J Immunol. 1984;19(6):575-9.

29. Sedgwick JD, Holt PG. Kinetics and distribution of antigen-specific lgEsecreting cells during the primary antibody response in the rat. J Exp Med. 1983;157(6):2178-83.

30. Holt PG, Sedgwick JD, O'Leary C, Krska K, Leivers S. Long-lived lgE- and lgGsecreting cells in rodents manifesting persistent antibody responses. Cell Immunol. 1984;89(2):281-9.

31. Mizutani Y, Shiogama K, Onouchi T, Sakurai K, Inada K, Tsutsumi Y. Enzymelabeled antigen method: development and application of the novel approach for identifying plasma cells locally producing disease-specific antibodies in inflammatory lesions. Acta Histochem Cytochem. 2016;49(1):7-19.

32. Mizutani $Y$, Tsuge $S$, Takeda H, Hasegawa Y, Shiogama $K$, Onouchi T, et al. In situ visualization of plasma cells producing antibodies reactive to Porphyromonas gingivalis in periodontitis: the application of the enzyme-labeled antigen method. Mol Oral Microbiol. 2014;29(4):156-73.

33. Pelletier N, McHeyzer-Williams LJ, Wong KA, Urich E, Fazilleau N, McHeyzerWilliams MG. Plasma cells negatively regulate the follicular helper T cell program. Nat Immunol. 2010;11(12):1110-8.

34. Shen P, Roch T, Lampropoulou V, O'Connor RA, Stervbo U, Hilgenberg E, et al. IL-35-producing B cells are critical regulators of immunity during autoimmune and infectious diseases. Nature. 2014;507(7492):366-70.

35. Wang RX, Yu CR, Dambuza IM, Mahdi RM, Dolinska MB, Sergeev YV, et al. Interleukin-35 induces regulatory B cells that suppress autoimmune disease. Nat Med. 2014;20(6):633-41.

36. Jing L, Kim S, Sun L, Wang L, Mildner E, Divaris K, et al. IL-37- and IL-35/ IL-37-producing plasma cells in chronic periodontitis. J Dent Res. 2019;98(7):813-21.

37. Shen P, Fillatreau S. Antibody-independent functions of B cells: a focus on cytokines. Nat Rev Immunol. 2015;15(7):441-51.

38. Mahanonda R, Champaiboon C, Subbalekha K, Sa-Ard-lam N, Rattanathammatada W, Thawanaphong S, et al. Human memory B cells in healthy gingiva, gingivitis, and periodontitis. J Immunol. 2016;197(3):715-25.

39. Han Y, Jin Y, Miao Y, Shi T, Lin X. Improved RANKL expression and osteoclastogenesis induction of CD27+CD38- memory B cells: a link between $B$ cells and alveolar bone damage in periodontitis. J Periodontal Res. 2019:54(1):73-80.

40. Murray PJ, Allen JE, Biswas SK, Fisher EA, Gilroy DW, Goerdt S, et al. Macrophage activation and polarization: nomenclature and experimental guidelines. Immunity. 2014;41(1):14-20.

41. Sima C, Glogauer M. Macrophage subsets and osteoimmunology: tuning of the immunological recognition and effector systems that maintain alveolar bone. Periodontology 2000. 2013;63(1):80-101.

\section{Publisher's Note}

Springer Nature remains neutral with regard to jurisdictional claims in published maps and institutional affiliations. 